\title{
Amplitude detection of weak periodic signal based on chaos and multiple autocorrelation
}

\author{
Yun $\mathrm{Li}^{1, \mathrm{a}}$, Siming Wang ${ }^{1, \mathrm{~b}}$ and Kang $\mathrm{Li}^{2, \mathrm{c}}$ \\ ${ }^{1}$ School of Automation\&Electrical Engineering, Lanzhou Jiao Tong University,Lanzhou \\ 730070,China; \\ ${ }^{2}$ Jinan railway administration section of jinan Qingdao motor car motor train-set,Jinan \\ 250000, China.

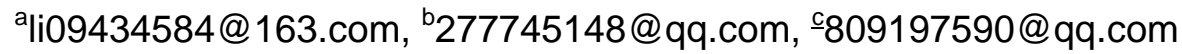

Keywords: Chaotic systems, Weak sine signal, Duffing chaotic oscillator, multiple autocorrelation algorithm, Amplitude detection.

\begin{abstract}
It is introduced the basic principle of weak periodic signal detection based on chaotic vibration subsystem. The shortcomings of the chaotic Duffing oscillator are presented by analyzingthe traditional detection results and detection principle based on Duffing oscillator, To make up the shortage of Duffing chaotic oscillator,it used multiple auto correlation algorithm to improve the signal to noise ratio of the system. Then the signal Then the output of the multiple autocorrelation algorithm is processed to improved chaotic oscillator system to detect the weak signal of arbitrary targets. Besides,to improve the detection accuracy of the system,the critical threshold of the system is determined by the method of recording the time points of the chaotic state.The detection results of the two methods are compared with the results of the methods, which are based on the detection of the Duffing oscillator and the multiple autocorrelation and the chaotic system,it can proved the feasibility and effectiveness of the two algorithms combined detection system.
\end{abstract}

\section{Introduction}

At present, we are in the information age, the information is the most important carrier of informationage, so the acquisition of information is an important issue in today's society.Signal detection has gradually become a hot research in the field,which is an important means of acquiring information.At this stage, the existing traditional and conventional detection methods are used,many of the objects(such as weak light signal, weak signal and weak current signal voltage signal) are not detected.So how to reduce the signal to noise ratio of input signal in strong noise background is an important research content in signal processing ${ }^{[1-4]}$.At present, many scholars have proposed some effective methods for weak signal detection,a large number of experimental studies have proved that the chaotic system has two characteristics, which are sensitive to initial value and immune to noise,so it gradually become the research hotspot in weak signal detection ${ }^{[5-6]}$.

\section{Signal detection method based on chaotic Duffing oscillator weak}

\subsection{Mathematical model of Duffing oscillator.}

The mathematical model of Duffing oscillator is ${ }^{[7]}$ :

$$
x(t)+k \dot{x(t)}-x(t)+x^{3}(t)=r \cos (t)(1)
$$

In the formula, $\left(-x(t)+x^{3}(t)\right)$ is the nonlinear restoring force of the chaotic system, $\mathrm{K}$ is the damping coefficient, $w$ is theangle frequency of driving force and $r$ is theamplitude ofdriving force of the Duffing oscillator.

In order to facilitate the detection of weak target signals of various frequencies, the state equation in the time scale is: 


$$
\left\{\begin{array}{l}
\dot{x}=\omega y \\
y=\omega\left(-k y+x-x^{3}+r \cos (\omega \tau)\right)
\end{array}\right.
$$

\subsection{Detection model and results based on chaotic Duffing oscillator.}

The mathematical model of Duffing equation in SIMULINK is established.In the model $\mathrm{k}=0.5, \mathrm{w}=1$,and the amplitude of the signal to be measured is 0.54 .First, the threshold value of the chaotic system which is from the chaotic state to the large scale periodic state is determined.According to the reference 1-3,In this paper,the threshold is 0.8343.Figure1 shows the system phase trajectories in the critical state of chaos.When the perturbation is different, the trajectory of the system is different.When the pure noise signal is added, there is no obvious change in the phase trajectories due to the strong immunity to noise signals,the phase trajectory diagram is shown in Figure2,the phase trajectory map is not smooth, but the shape is not changed;when signal to be mixed with noise signals is added,the phase trajectory of the system will be from the chaotic state to periodic state,because of the sensitivity of the chaotic system to the initial value of weak signal,the change of phase trajectory is obvious,the phase trajectory diagram is shown in Figure3.It can prove the existence of the periodic signal to be measured.

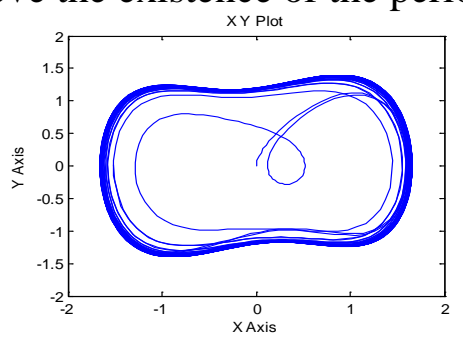

Fig.1

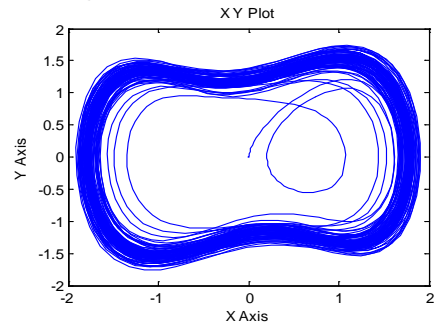

Fig.2

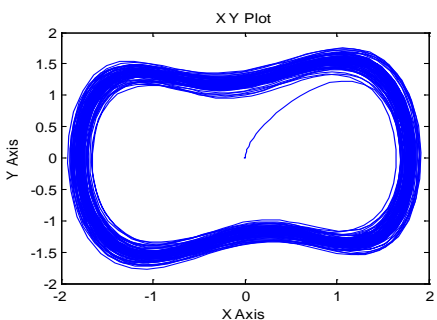

Fig.3

Fig.1Initial chaos critical state

Fig. 2 Phase trajectory after adding pure noise

Fig. 3 Phase trajectory after adding hybrid signal

\section{Determination of threshold of chaos system}

The threshold value of the traditional chaotic system is determined by direct observation method or by calculating the largest Lyapunov exponent. The first method has a large error and method two is too large to calculate.According to the time and the time required by the system to enter the chaotic state, the speed of the system to enter the state transition can be determined,based on this theory, the precise threshold of the systemcan be determined by the experimental method ${ }^{[10]}$. For a system in which it is in a chaotic state,the relationship between the output $\mathrm{x}$ and time $\mathrm{t}$ is observed by the oscilloscope,as shown in Figure4.

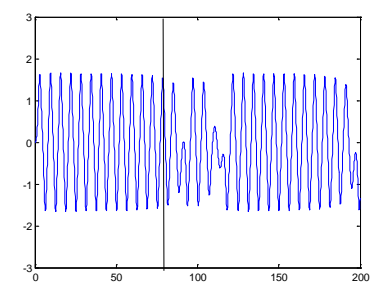

(a) $r=0.833, t=85$

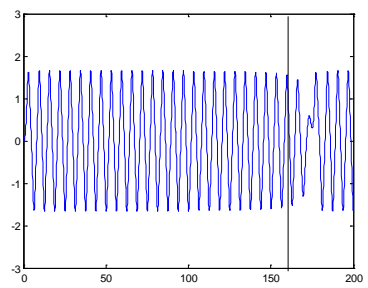

(d) $r=0.8342, t=183$

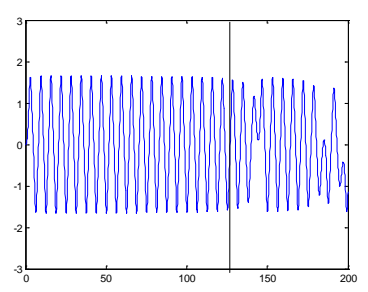

(b) $r=0.834, t=134$

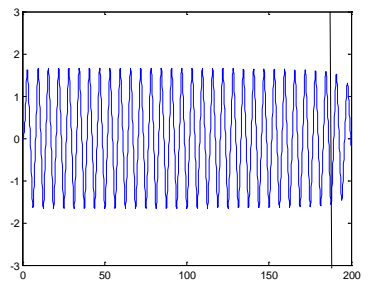

(e) $r=0.83422, t=196$

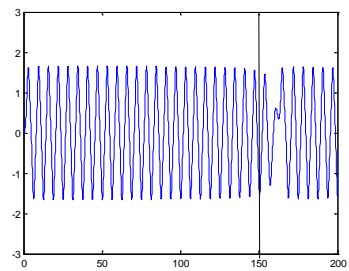

(c) $\mathrm{r}=0.8341, \mathrm{t}=150$

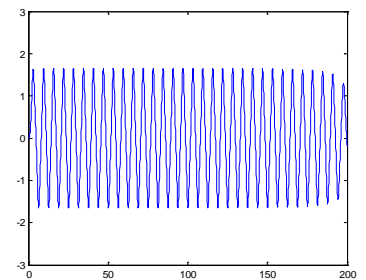

$(h) r=0.83423, t=199$

Fig. 4 The relationship between $t$ and time $x$ with the change of $r$ 
The periodic signal is turned into an irregular signal at a certain time,the time points and the driving force are recorded,the record data as shown in Table 1.The quadratic curve is carried on by least square method according these datas,the fitting results are shown in figure5.We can see that the original curve and the fitting curve tends to an asymptoter $=0.834225$.So the amplitude corresponding to the asymptote is the critical threshold of chaotic system.In this paper,to improve the detection accuracy of the system,we use the method to determine the threshold value of the system..

Table 1 The relationship between system driving force amplitude $\mathrm{r}$ and time $\mathrm{t}$

\begin{tabular}{c|c|c|c|c|c|c|c|c|c|}
\hline 序号 & 1 & 2 & 3 & 4 & 5 & 6 & 7 & 8 & 9 \\
\hline $\mathrm{r}$ & 0.831 & 0.832 & 0.833 & 0.834 & 0.8341 & 0.8342 & 0.83421 & 0.83422 & 0.834225 \\
$\begin{array}{c}\text { 时间 } \\
/ \mathrm{s}\end{array}$ & 60 & 73 & 92 & 143 & 151 & 186 & 190 & 196 & $\infty$ \\
\hline
\end{tabular}

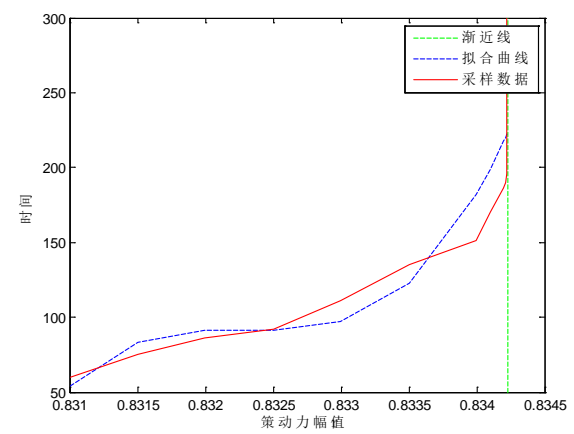

Fig. 5 The fitting results

\section{Detection principle and results of the algorithm based on chaotic oscillator and multiple self correlation algorithm}

\subsection{Auto correlation algorithm.}

The working principle of weak signal detection based on auto correlation algorithm is that it made a calculation for the input signal and the delay of the input signal by using the random noise to eliminate the noise signal,so as to improve the signal to noise ratio.The input signal for the auto correlator is

$$
F(t)=s(t)+n(t)=f[\cos (\omega t)+\varphi]+n(t)
$$

Its auto correlation function is

$$
\begin{aligned}
& R(\tau)=E[f(t) f(t+\tau)]=R_{S}(\tau) \\
& E[s(t) \circ n(t+\tau)]+E[s(t+\tau) \circ n(t)]+R_{N}(\tau)
\end{aligned}
$$

The auto correlation function of the target signal is

$$
\begin{aligned}
& R_{S}(\tau) \cong \hat{R}_{S}(\tau)=\frac{1}{T-\tau} \int_{0}^{T-\tau} s(t) s(t+\tau) d t \\
& =\frac{1}{T-\tau} \int_{0}^{T-\tau} \frac{A^{2}}{2} \cos [\omega(2 t+\tau)+2 \varphi] d t+\frac{A^{2}}{2} \cos (\omega t)
\end{aligned}
$$

Auto correlation function of noise signal $R_{N}(\tau)$ is a function of the $\tau$, it tends to 0 with the increase of time. Therefore, in the actual detection and simulation,we made $R_{N}(\tau)=0$.

\subsection{Multipleauto correlation algorithm.}

According to the above analysis,we put the formula(6)(7) into the formula(2) to simplified and increase the integration time to the period T.The auto correlation function of the simplified model is shown in the formula (8).

$$
R_{F}(\tau)=\frac{A^{2}}{2} \cos (\omega \tau)+\frac{A^{2}}{2 T} \int_{0}^{T} \cos [\omega(2 t+\tau)+2 \varphi] d t+\frac{1}{T} \int_{0}^{T} s(t) d t \circ \frac{1}{T} \int_{0}^{T} n(t+\tau) d t+\frac{1}{T} \int_{0}^{T} s(t+\tau) d t \circ \frac{1}{T} \int_{0}^{T} n(t) d t+R_{N}(\tau)
$$


Rewrite as

$$
F_{1}(t)=f_{1} \cos \left(\omega_{1} t+\varphi_{1}\right)+n_{1}(t)
$$

The multiple auto correlation algorithm is that the F1 in the formula (7) is used as the F to calculate the autocorrelation. The two input of the auto correlator is the delay signal $f(t+\tau)$ and the original signal $f(t)$ respectively,we made a convolution calculation and integral,the output is auto correlation function $R(\tau)$. Repeat the above steps, we can complete auto correlation operation of the signal to be measured.The time sequence diagram of the signal to be measured with noise is as figure6(a),time series waveform chart of the signal to be measured after a self correlation function operation is as figure6(b),time series waveform chart of the signal to be measured after four self correlation function operation is as figure6(c).From the waveform of the signal can be seen that the signal is also affected by the noise.

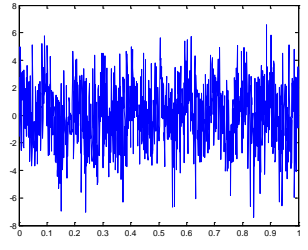

(a)

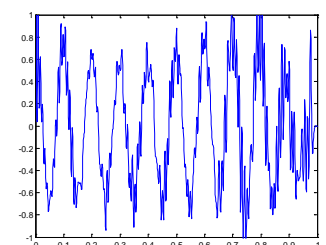

(b)

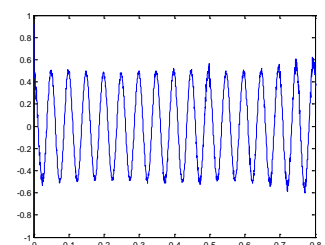

(c)

Fig. 6 Signal waveform to be measured

\subsection{Weak signal amplitude detection based on multiple auto correlation function and chaotic} oscillator.

The mathematical model of the traditional Duffing oscillator is improved,which is as follow.

$$
\left\{\begin{array}{l}
\dot{x}=\omega y \\
\dot{y}=\omega\left(-k y+x^{3}-x^{5}+K(a \cos (\omega t)+n(t))\right)
\end{array}\right.
$$

$\mathrm{K}$ is a amplifier.

To make the system in the critical state of chaos, $K$ is monitored.At this point $K_{d}{ }^{*} a=r_{d}$,according to this formula,we can calculate the signal amplitude a.According to the formula (8),a weak periodic signal detection system model is made which is based on multiple self correlation algorithm and chaotic Duffing oscillator system, $\mathrm{k}=0.5, \omega=1, \mathrm{r}_{\mathrm{d}}=0.834225$. The amplitude ofthe signal to be measured is $\mathrm{a}=0.54$. The noise signal is Gauss white noise.In the system,when $\mathrm{K}=1.3073^{*} 10^{8}$, the trajectory of the system has changed,which is from the chaotic state (Figure7 (a)) to the large scale periodic state (Figure7 (b)).It can be calculated that amplitude of periodic signal is 0.54000553 ,and the relative error of amplitude detection is $0.001024 \%$.So the detection error is small and the detection accuracy is higher.
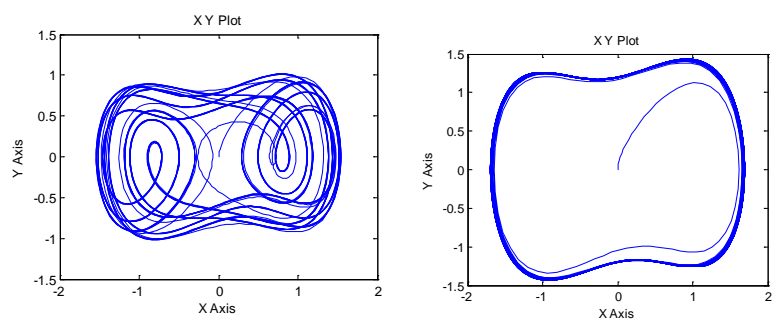

(a)Chaos state (b)Large scale periodic state

Fig. 7 Phase trajectory of detection system based on hybrid algorithm

\section{Summary}

Through the above analysis and experiment,it can be seen that the method can effectively improve the signal to noise ratio which combines the multiple autocorrelation algorithm and the Duffing oscillator detection,and detection accuracy has been greatly improved.According to the 
detection algorithm,the weak signal can be detected when the error level is above $10^{-5}$.The signal to noise ratio of the system is

$$
S N R=10 \lg \left(P_{S} / P_{N}\right)=-40.141 \mathrm{~dB}
$$

Compared with literature 8,9 and 10,the detection accuracy of the detection system can be improved by an order of magnitude in the case of the same signal to noise ratio,and the detection system can significantly improve the signal to noise ratio in the case of the detection of the same magnitude.

\section{References}

[1]. Chunyan Nie.Chaotic system and weak signal detection.Tsinghua University press,2009, p.41-124.

[2]. Jinzhan Gao.Weak signal detection.Tsinghua University press,2011, p.258-286.

[3]. Jin Tian,Hua Zhang.Statistical approach to weak signal detection and estimation using Duffing chaotic oscillators.SCIENCE CHINA.Vol.54(2011)No.11,p. 2324-2337.

[4]. Yunhong Xi,Lingzhi Xue.The Study for the Method to Weak Signal Detection Based on the Combination of the Chaotic.Scientific Journal of Information Engineering.Vol.3(2014)No.4,p.44-56.

[5]. Wang Yuegang,Wen Chaobin,Yang Jiasheng,et al.Adaptive control of chaos system based on the model free method.Journal of Physics.(2013)No.10,p.73-79.

[6]. Fan Jian,Zhao Wenli,Wang Wanqiang.Study on chaotic detection performance of weak periodic signal based on Duffing oscillator.Journal of Physics.(2013)No.18,p.54-59.

[7]. Gao Shilong,Zhong Suchuan.Weak signal detection based on chaos and stochastic resonance.Journal of Physics.(2012)No.18,p.41-48.

[8]. Wu Yongfeng,Huang Shaoping,Jin Guobin.Detection method of partial discharge signal based on coupled Duffing oscillator.Journal of Physics.(2013)No.13,p.132-139. 